\title{
On Axon Delay Functions and Spiking Activity
}

\author{
Conrad Attard and Andreas A. Albrecht
}

\begin{abstract}
Over the past few years, the importance of axonal conduction delays has been emphasized by a number of authors. Different models are proposed for the approximation of signal delays, where some of them have been evaluated in the context of the optimal neuronal layout problem. Our paper presents computational experiments on the impact of two wiring cost functions, proposed by D. Chklovskii and O. Shefi et al., when applied to interneuronal connections in small ML neuronal networks.
\end{abstract}

\section{INTRODUCTION}

$\mathbf{T}$ HE simulation of networks of spiking neurons is a central issue in neuroscience. A recent overview and rigorous evaluation of different models of spiking neurons can be found in [7]. E.M. Izhikevich discusses twenty of the most prominent features of biological spiking neurons (tonic spiking, phasic spiking, threshold variability, etc.) against the expressiveness of eleven models of spiking neurons, including an assessment of the "biological plausibility" of neuron models. The author highlights three models as particularly suited for simulations of networks of spiking neurons, where the high degree of approximation of biophysical properties plays an important role. The three models are the HodgkinHuxley model, the Morris-Lecar model, and a new model developed by the author himself. Due to the high accuracy of the Hodgkin-Huxley model (four defining equations with tens of parameters), the model is computationally expensive and allows the simulation of small networks only with currently available hardware. This is why, at the moment, the Morris-Lecar model (two defining equations and a moderate number of parameters) is very popular in the computational neuroscience community.

As pointed out in [8], in most of the research on networks of spiking neurons synchrony of firing is emphasized, i.e. if two or more neurons have a common post-synaptic target and fire synchronously, then their spikes arrive at the target at the same time, thereby evoking potent post-synaptic responses. This implies an implicit assumption that the axonal conduction delays are negligible or equal. E.M. Izhikevich [8] (see also [9]) investigates the effect of signal delays and pre-synaptic (asynchronous) firing sequences on postsynaptic responses, i.e. different (initial) pre-synaptic firing sequences result in the activation of different subsets of neurons. The underlying approximation of neuronal activity is the model introduced in [7], which comprises of three variables and four parameters. To distinguish the effect from random asynchronous firing, the notion of "polychronization" is introduced, and subsets of activated neurons are

Conrad Attard and Andreas A. Albrecht are with the School of Computer Science, University of Hertfordshire, Hatfield, Herts AL10 9AB, UK (email: \{C.Attard, A.Albrecht\}@ herts.ac.uk). called "polychronous groups." For constant, randomly chosen conduction delays, results from simulations of a $10^{3}$ neuronal network are presented and discussed in [8]. Polychronous groups are identified from their topological relation and firing activity in simulations after convergence of the network. The author identifies 5,269 different polychronous groups in the network of $10^{3}$ neurons, which suggests an unprecedented memory capacity of such systems. A natural extension of this work is to investigate specific cost functions assiciated with conduction delays, instead of constant, randomly chosen values. The present paper presents preliminary research into this direction. A future goal is to optimize the 2D and 3D placement of neurons in such a way that the number of polychronous groups is maximized.

\section{Modelling CONDUCTION DELAYS}

Various models have been proposed recently for numeric simulations of axonal conduction delays; see [1], [2], [3], [4], [13] and the literature therein. We intend to employ the connection cost function as proposed in [2], [3], [4] and in [13], since the authors provide a justification of their model that is based on experimental evidence in the context of the Optimal Neuronal Layout Problem.

\section{A. The Chklovskii Delay Function}

A common assumption is that wiring costs are related to the wiring volume. However, in his paper [4], D. Chklovskii attempts to tackle the problem of wiring costs in neuronal networks by proposing a cost function that is determined by the square of the wire length. A volume cost function would result in axons tending to be extremely thin. Although this may decrease energy and other requirements, it would certainly hinder the propagation of signals. These conflicting requirements tend to suggest some optimum combination that must be factored in any plausible solution.

The Chklovskii model is motivated by a comparison of results from neuronal layout calculations to the arrangements in the macaque pre-frontal cortex and the Caenorhabditis elegans, in the latter case for 279 neurons [2], [3]. The experimental analysis suggests that wiring costs are indieed proportional to the "wire volume" for a fixed diameter, and the costs grow linearly with the wire length. Furthermore, the wiring cost is a function of the propagation delay $T=L / k \cdot d$, where $L$ is the length of the connection and $k \cdot d$ is the signal speed for the diameter $d ; k=$ const. This implies the approximation

$$
\text { Cost }=\alpha \cdot \frac{\pi}{4} \cdot d^{2} \cdot L+\beta \cdot\left(\frac{L}{k \cdot d}\right)^{\gamma},
$$

where $\alpha, \beta, \gamma=$ const. If the cost function is minimized with respect to the diameter, i.e. $\partial$ Cost $/ \partial d=0$, one obtains 
Cost $\asymp L^{3 \cdot \gamma /(\gamma+2)}$, which means Cost $\asymp L$ for $\gamma=1$ and Cost $\asymp L^{3}$ as $\gamma \rightarrow \infty$. As a compromise, Cost $\asymp L^{2}$ is assumed in [4]. One of the main objectives of the present paper is to analyse whether this assumption is justified, where the basic parameter is the intensity of spiking activities of neurons in the Morris-Lecar model.

\section{B. The Shefi et al. Delay Function}

In [13], Shefi et al. propose an alternative approach to the optimal layout problem and the laws that dictate the formation of neural structures, drawing on the earlier works of C.D. Murray [11], [12]. Murray's papers originally dealt with volumes and the relationship between the diameters of a given pair of sub-branches of a blood vessel or tree. This relationship basically stated that the optimal diameters of the bifurcating branches depended on the diameter of the parent branch or blood vessel and the angle between them, given by the following equations:

$$
\begin{aligned}
\cos \left(\alpha_{1}\right) & =\frac{d_{0}^{4}+d_{1}^{4}-d_{2}^{4}}{2 d_{1}^{2} \cdot d_{0}^{2}}, \\
\cos \left(\alpha_{2}\right) & =\frac{d_{0}^{4}+d_{2}^{4}-d_{1}^{4}}{2 d_{2}^{2} \cdot d_{0}^{2}}, \\
\cos \left(\alpha_{1}+\alpha_{2}\right) & =\frac{d_{0}^{4}+d_{1}^{4}-d_{2}^{4}}{2 d_{1}^{2} \cdot d_{2}^{2}},
\end{aligned}
$$

where $d_{0}$ is the parent neurite diameter, $d_{1}$ and $d_{2}$ are the child neurite diameters, and $\alpha_{1}$ and $\alpha_{2}$ are the angles between the central axis of the parent neurite and their respective child neurites.

Complimentary to the diameter and angles relationship, Shefi et al. [13] considered the balance of tensions between the main neurite and the sub-neurites. This ensures the optimal layout and diameters and is mathematically equivalent to Murray's equations. However, for the scope of this paper we shall limit ourselves to (2) until (4). Both Murray's and tension approaches were applied to data from cultured networks of locust ganglia [13]. Although neither was conclusive, results were overall satisfactory for both models [13].

For the axon delay experiments described in this paper, the total wiring $\operatorname{cost} \varphi$ for each neurite shall be taken as the volume of the parent neurite up to the bifurcation point added to that of the child neurite as suggested in [13]:

$$
\varphi=\pi \cdot \frac{\left(d_{0}^{2} \cdot l_{0}+d_{1}^{2} \cdot l_{1}\right)}{4},
$$

where $d_{0}$ and $d_{1}$ respectively represent the parent and child neurite diameters and $l_{0}$ and $l_{1}$ are the parent and child "wire" lengths.

\section{THE NEURONAL NETWORK}

The networks devised for the experiments are based on the assumption that neurons in nature tend to communicate largely through spike trains. The network simulation time is divided into iterations, where during an iteration every neuron can simulate the impact of the efferent spikes on its membrane and generate its own action potentials as a result. These new potentials are propagated at the end of the current iteration but will only have effect in the next one. All spikes are stored and processed in order of arrival at the neuron. This strategy, although artificial, ensures that all spikes generated in the network will be processed in a synchronised manner, which does not affect the study of the axon delays.

\section{A. Network for Chklovskii Simulations}

The physical design for the Chklovskii experiment network is shown in Fig. 1. The 24 ML-type neurons are linked in an arbitrary random manner. Consequently, a spike generated by one particular neuron is transmitted to a few (1 to 2) other neurons. Although the amplitude for a give spike will be the same for all, the arrival time will vary depending on the axon length.

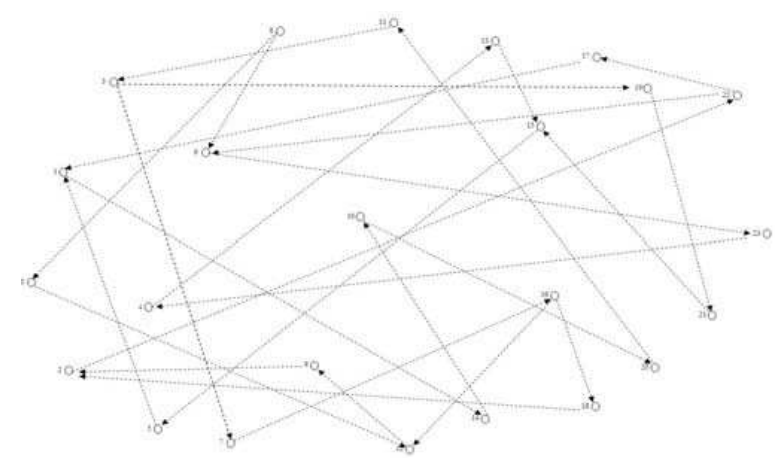

Fig. 1. Layout for Chklovskii simulations.

\section{B. Network for Shefi et al. Simulations}

A different approach is taken for the Shefi experiment where the neural connections, as seen in Fig. 2, follow a pattern dictated largely by the bifurcations of the axons. In this case the axon delay is calculated by the total axon-neurite volume.

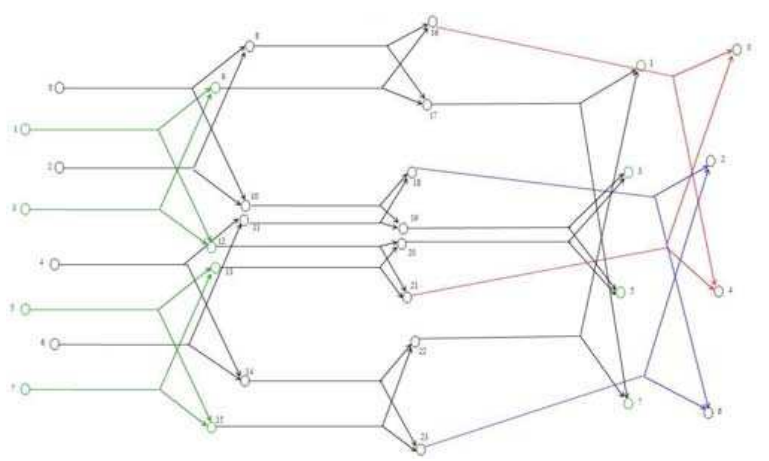

Fig. 2. Network diagram for Shefi et al. simulations. 
Depending on the cost function applied, axon length or volume directly affects the arrival of a pulse transmitted from one neuron to another.

\section{COMPutational EXPERIMEnts}

The simulation and integration of the ML equations is handled by the XPPAUT program package. This freeware, written by G.B. Ermentrout [5], is a graphical tool that we utilized for solving the ML's differential equations, thus allowing each neuron to change its state in accordance with the input spikes it receives, see also [6], [10].

\section{A. The Morris-Lecar Model}

For each node of the network, XPPAUT [5] must be presented with a file known as an ODE containing the model description and all the necessary parameters. Thus, the file consists of equations, XPPAUT functions and data variables.

The following excerpt, taken from an ODE file for a single neuron model (number zero), lists the first eight lines. It shows the ML equations and the the initial values for $V$ and $w$ which are held in each MLNeuron object of the simulation.

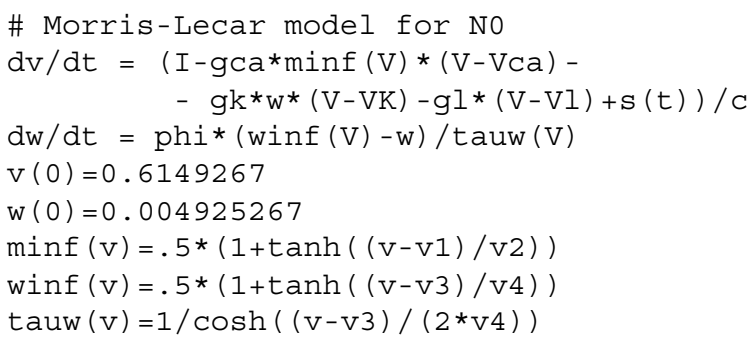

The next part is taken from the same ODE file and consists entirely of parameters representing the external current, ionic conductances, voltages and the value for $\phi$. The pair of ' $t$ " values denotes the start and the end time for the spikes. The ' $s$ ' values represent the amplitude for each spike.

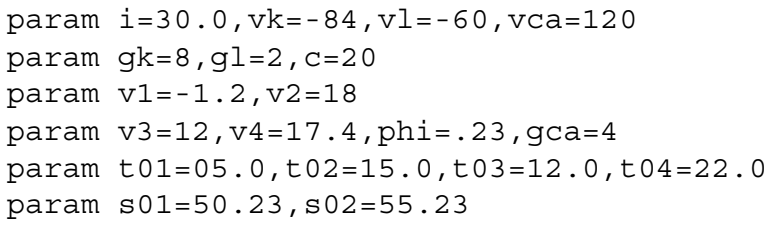

The last part of the ODE file shows the heavyside function used to simulate the spiking action. The lines two and three define the output file name, the duration of the integration and the minimum time units both in $m s$.

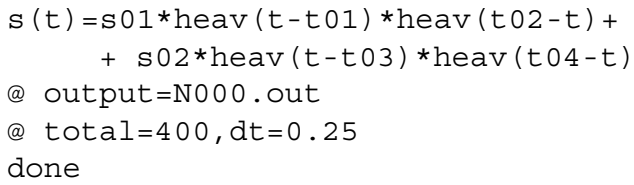

During the processing of its new state, a neuronal node of the network prepares an ODE file similar to the one just described. Parameters such as ionic conductances are left unchanged and are common to all the neurons in the network.
On the other hand, the neuron's current values of $V$ and $w$ and the amplitudes, offsets and durations of any spikes to be applied are set individually for each neuron. The time window for a neuron to run is set at $400 \mathrm{~ms}$. XPPAUT is run in quiet mode (non graphical) with the ODE file passed as a run-time parameter. This creates an output file of the resulting integration, listing the values of $V$ and $w$ for the $400 \mathrm{~ms}$ time window.

The following parameters are common to all ML neurons: $\mathrm{vk}=-84 \mathrm{mV}$ : Potassium conductance potential; $\mathrm{vl}=-60 \mathrm{mV}$ : Leak conductance potential; vca $=120 \mathrm{mV}$ : Calcium conductance potential; $\mathrm{gk}=8$ : Conductance value for Potassium; $\mathrm{gl}=2$ : Conductance value for Leak; gca=4: Conductance value for Calcium; $\mathrm{c}=20 \mu \mathrm{F}:$ Membrane capacitance;

v1=-1.2mV: Potential at which $M_{\infty}=0.5$; v2=18: $\frac{1}{M_{\infty}(V)}$;

v3=12: Potential at which $N_{\infty}=0.5$;

v4=17.4: $\frac{1}{N_{\infty}(V)}$;

$\phi=.23$ : Temperature constant;

$\mathrm{I}=30 \mu \mathrm{A}$ : Applied current.

In each experiment, we executed 14 iterations with each iteration simulating $400 \mathrm{~ms}$, i.e. the total simulation time of a single experiment was $5,600 \mathrm{~ms}$. The settings were the results of preliminary experiments with the XPAUT program package.

\section{B. Chklovskii Wiring Cost Results}

As mentioned in Section II-A, the main concern was to analyse the impact of the power $\gamma$ in (1) on the spiking activity. Six separate experiments were conducted on the same network of 24 Morris-Lecar neurons for the Chklovskii wiring cost accoding to Cost $\asymp L^{3 \cdot \gamma /(\gamma+2)}=L^{p}$ and variable $p$ (actually, $\gamma$ ). The settings for $p$ were $p=0.0$ (no wiring cost), 1.0, 1.5, 2.0, 2.5 and 3.0.

Each set of six experiments was conducted with four different initial spike sets. The sets consist of mixed patterns of repeated 3,2 , or single spikes.

Below are the results for the four initial spike sets.

\section{Chklovski Cost Function: Spike Set 1}

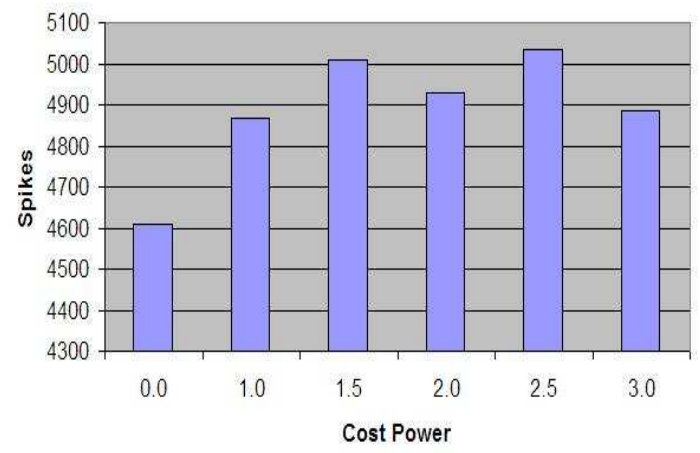

Fig. 3a. Number of spikes produced for spike set 1 . 
Chklovski Cost Functon: Spike Set 2

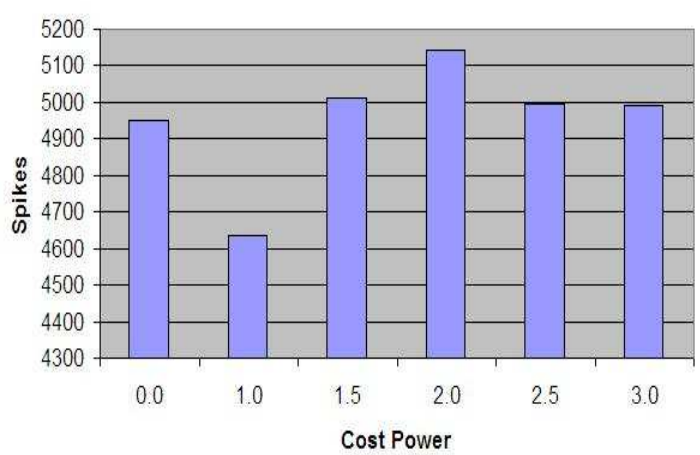

Fig. 3b. Number of spikes produced for spike set 2 .

\section{Chklovskii Cost Function: Spike Set 3}

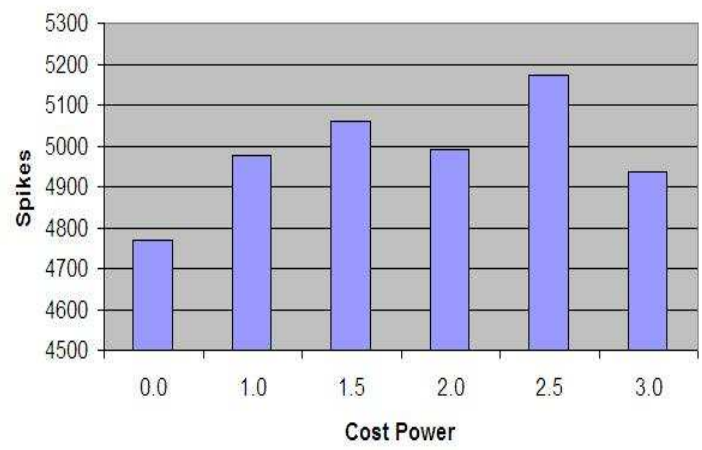

Fig. 3c. Number of spikes produced for spike set 3 .

\section{Chklovskii Cost Function: Spike Set 4}

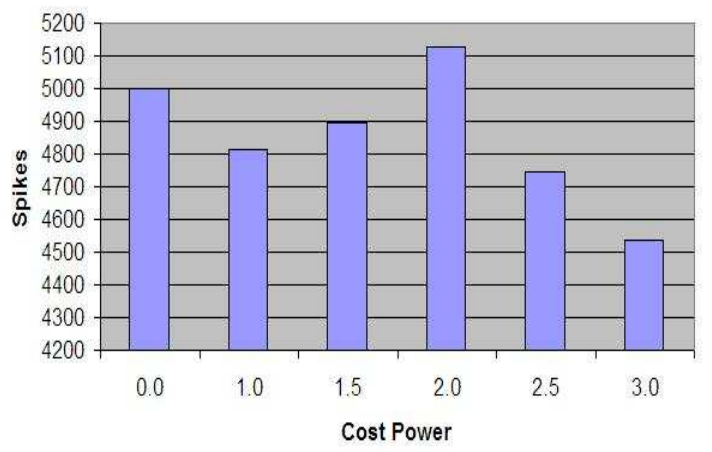

Fig. 3d. Number of spikes produced for spike set 4 .

As can be seen from Fig. 3a until Fig. 3d, values $p \in$ $\{1.5,2.0,2.5\}$ produce the largest number of spikes, i.e. at least one of the values, but not necessarily for $p=2.0$. A different picture emerges, if the average spiking activity is calculated over the four initial spike sets. Fig. 4 clearly indicates the highest average spiking activity for Cost $\asymp L^{2}$.

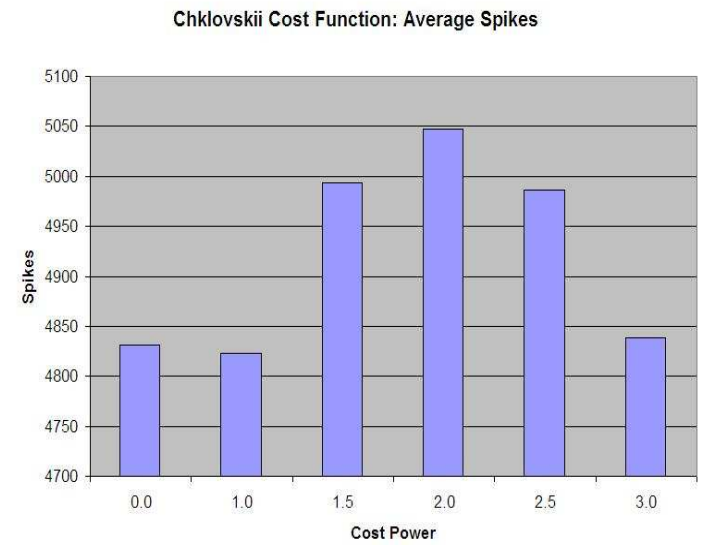

Fig. 4. Average number of spikes for network from Fig. 1.

\section{Shefi et al. Wiring Cost Results}

Two simulations were conducted to demonstrate the impact of the Shefi et al. cost function: one simulation with cost function $\varphi$ from (5), and one with conductance delay equal to zero. The same four sets of initial spikes were used as in the Chklovskii simulations.

In all four sets of experiments, the spiking activity with conduction delay was much higher when compared to the zero-cost case. We present the two experiments with the largest and the smallest difference in spiking activities only:

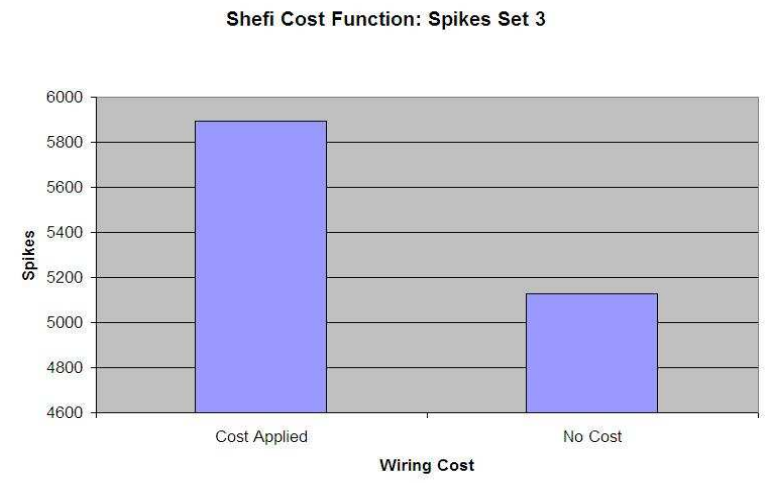

Fig. 5a. Number of spikes produced for spike set 3 .

Shefi Cost Function: Spikes Set 4

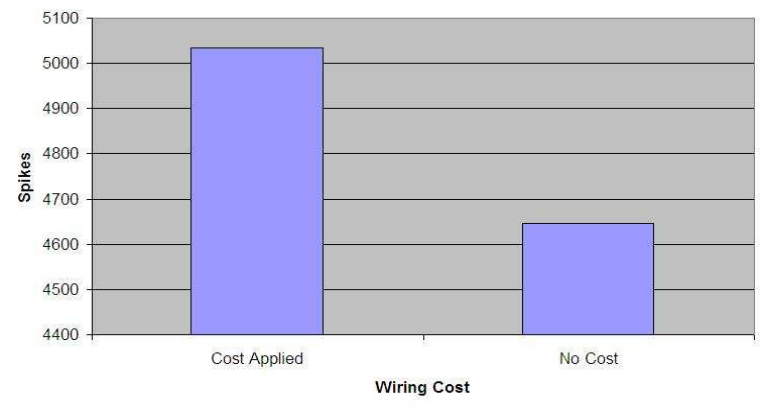

Fig. 5b. Number of spikes produced for spike set 4 . 
Fig. 6 shows the significant difference between the two cases for the average values over all four initial spike sets.

Shefi Cost Function: Average Spikes

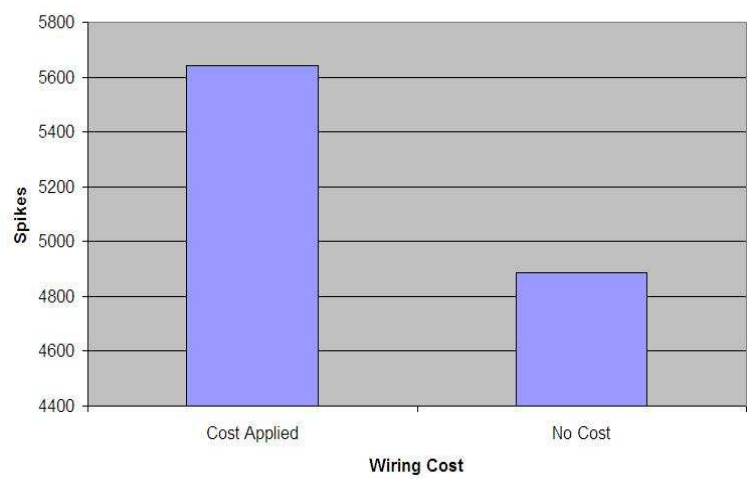

Fig. 6. Average number of spikes for network from Fig. 2.

When comparing the Chklovskii delay model and the Shefi et al. cost function, we note that for different topologies, but the same number of ML neurons and the same simulation time, the average number of spikes produced within the 14 iterations of equal length differs only marginally.

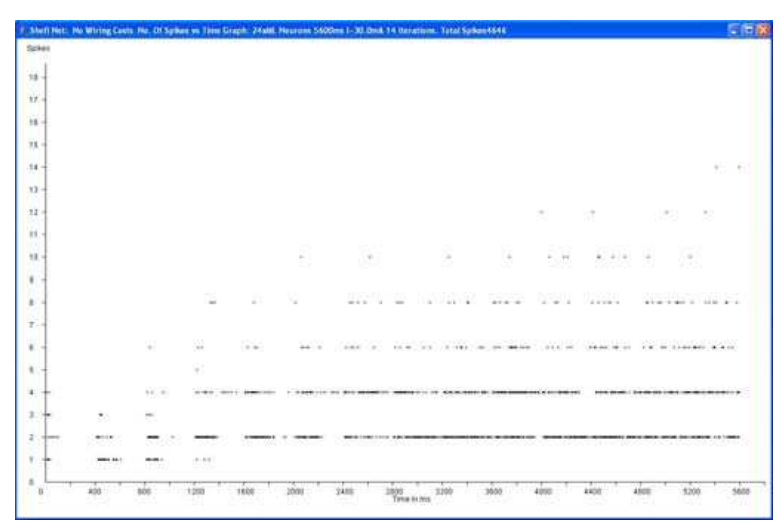

Fig. 7a. Spikes produced vs. time for zero wiring costs.

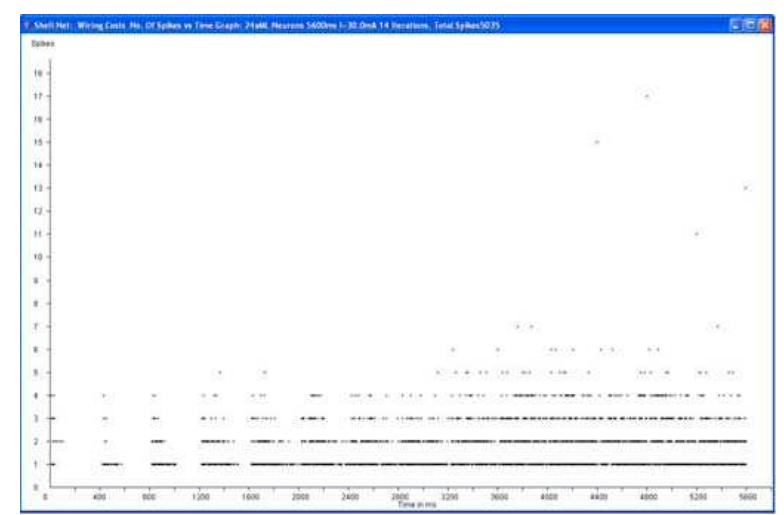

Fig. 7b. Spikes produced vs. time with wiring costs.

In Fig. 7a and Fig. $7 \mathrm{~b}$ we present the spiking activity vs. simulation time for the Shefi et al. model and the initial spike set no. 4. We note that from $\mathrm{T}=1800 \mathrm{~ms}$ onwards the network with the Shefi et al. volume cost function is spiking with growing frequency, see Fig. 7b. The other network (without wiring cost, Fig. 7a), increases intensity of spiking activity largely between $\mathrm{T}=2400 \mathrm{~ms}$ and $\mathrm{T}=2800 \mathrm{~ms}$. Furthermore, most common spike bursts for the wiring cost network are between 1 and 3 .

Curiously, the network with zero wiring cost tends to produce many even numbered and more scattered bursts of around 2 to 6 spikes. This might be caused by the bifurcating layout of the Shefi et al. network (see Fig. 2). When the wiring cost is applied, the differences in angle and volume of the dendrites desynchronise the spikes propagated to the efferent neurons: let us consider a neuron A that is connected to neurons $\mathrm{B}$ and $\mathrm{C}$ through a bifurcation in the axon where volume $\mathrm{A} \rightarrow \mathrm{B}$ is greater than $\mathrm{A} \rightarrow \mathrm{C}$. Consequently a spike from $\mathrm{A}$ would be propagated faster to $\mathrm{C}$ than to $\mathrm{B}$, thus affecting the transfer of this potential through the network. When no costs are applied, the spike is propagated simultaneously to both and also affects the efferent neuron at the time. If both efferent neurons are both roughly in the same excited state, there is a high probability that both will propagate similar spikes. This dual propagation seems to produce even numbers of spikes.

\section{REFERENCES}

[1] R.E. Burke, "Comparison of alternative designs for reducing complex neurons to equivalent cables," Journal of Computational Neuroscience, vol. 9, pp. 31-47, 2000.

[2] B.L. Chen and D.B. Chklovskii, "Placement and routing optimization in the brain," Proceedings of the International Symposium on Physical Design, pp. 136-141, 2006.

[3] B.L. Chen, D.H. Hall and D.B. Chklovskii, "Wiring optimization can relate neuronal structure and function," Proceedings of the National Academy of Sciences of the United States of America, vol. 103, no. 12, pp. 4723-4728, 2006.

[4] D.B. Chklovskii, "Exact solution for the optimal neuronal layout problem," Neuronal Computation, vol. 16, pp. 2067-2078, 2004.

[5] G.B. Ermentrout, XPPAUT, http://www.math.pitt.edu/ bard/xpp/.

[6] B.S. Gutkin and G.B. Ermentrout, "Dynamics of membrane excitability determine interspike interval variability: A link between spike generation mechanisms and cortical spike train statistics," Neural Computation, vol. 10, pp. 1047-1065, 1998.

[7] E.M. Izhikevich, "Which model to use for cortical spiking neurons?," IEEE Transactions on Neuronal Networks, vol. 15, no. 5, pp. 10631069, 2004.

[8] E.M. Izhikevich, "Polychronization: Computation with spikes," Neural Computation, vol. 18, no. 2, pp. 245-282, 2006.

[9] E.M. Izhikevich, Dynamical systems in neuroscience: The geometry of excitability and bursting, MIT Press, 2007.

[10] C. Morris and H. Lecar, "Voltage oscillations in the barnacle giant muscle fiber," Biophysical Journal, vol. 35, pp. 193-213, 1981.

[11] C.D. Murray, "The physiological principle of minimum work. I. The vascular system and the cost of blood volume," Proceedings of the National Academy of Sciences of the United States of America, vol. 12, no. 3, pp. 207-214, 1926

[12] C.D. Murray, "A relationship between circumference and weight in trees and its bearing on branching angles," The Journal of General Physiology, vol. 10, pp. 725-729, 1927.

[13] O. Shefi, A. Harela, D.B. Chklovskii, E. Ben-Jacobb and A. Ayali, "Biophysical constraints on neuronal branching," Neurocomputing, vol. 58-60, pp. 487-495, 2004. 